\title{
Quenched dynamics of artificial colloidal spin ice
}

\author{
A. Libál $\odot,{ }^{1,2,3}$ A. del Campo $\odot,,^{2,4,5,6}$ C. Nisoli, ${ }^{2,3}$ C. Reichhardt, ${ }^{2,3}$ and C. J. O. Reichhardt $\odot^{2,3}$ \\ ${ }^{1}$ Department of Mathematics and Computer Science, Babeş-Bolyai University, Cluj-Napoca, Cluj 400084, Romania \\ ${ }^{2}$ Theoretical Division, Los Alamos National Laboratory, Los Alamos, New Mexico 87545, USA \\ ${ }^{3}$ Center for Nonlinear Studies, Los Alamos National Laboratory, Los Alamos, New Mexico 87545, USA \\ ${ }^{4}$ Donostia International Physics Center, 20018 San Sebastián, Spain \\ ${ }^{5}$ IKERBASQUE, Basque Foundation for Science, 48013 Bilbao, Spain \\ ${ }^{6}$ Department of Physics, University of Massachusetts, Boston, Massachusetts 02125, USA
}

(Received 6 September 2019; accepted 30 July 2020; published 17 September 2020)

\begin{abstract}
Artificial spin ices are ideal frustrated model systems in which to explore or design emergent phenomena with unprecedented characterization of the constituent degrees of freedom. In square spin ice, violations of the ice rule are topological excitations essential to the kinetics of the system, providing an ideal test bed for studying the dynamics of such defects under varied quench rates. In this work we examine possible scenarios including the Kibble-Zurek mechanism and critical coarsening in colloidal square and hexagonal ice under quenches from a weakly interacting liquid state into a strongly interacting regime. As expected, for infinitely slow quenches, the system is free of defects such as monopoles, while for increasing quench rate, an increasing number of defects in the form of monopoles or grain boundaries remain in the sample. For square ice, we find regimes in which the defect population decreases as a power law with decreasing quench rate. A detailed scaling analysis shows that for a wide range of parameters, including quench rates that are accessible by experiments, the behavior is best described by critical coarsening rather than the Zurek-Kibble scenario if we assume that the equilibrium phase transition in this system is in the Ising universality class. The appearance of critical coarsening is likely due to the strong defect interactions in the colloidal ice system leading to relevant defect dynamics during the quench. For hexagonal ice we do not find evidence for a power-law decay in the defect density, which is consistent with the absence of an equilibrium phase transition in the hexagonal ice as well as a lack of critical coarsening.
\end{abstract}

DOI: 10.1103/PhysRevResearch.2.033433

\section{INTRODUCTION}

The term artificial spin ice (ASI) describes a variety of systems that can be modeled by frustrated, interacting effective binary degrees of freedom which obey the ice rule. The ASI size scales are much larger than those of molecular and atomic spin ice systems, allowing the individual spin degrees of freedom to be imaged directly [1-5]. Artificial spin ice can be realized using arrays of nanomagnets [1-3,611], colloids in ordered trap arrays [12-16], and vortices in nanostructured superconductors [17-21]. Of the wide variety of different ASI geometries, the first and most studied are the square $[1-3,10,22]$ and hexagonal ices [2,6-9,13,20,23]. While both of these geometries obey the ice rule in their low-energy states, the square ice exhibits an antiferromagnetic long-range-ordered ground state, while the hexagonal ice does not have long-range order but can form ice-ruleobeying states.

Published by the American Physical Society under the terms of the Creative Commons Attribution 4.0 International license. Further distribution of this work must maintain attribution to the author(s) and the published article's title, journal citation, and DOI.
A particularly appealing feature of ASI systems is that they contain well-defined defects that take the form of nonice-rule-obeying vertices which can be imaged directly in experiment. In traditional spin ice systems on the molecular scale, such imaging is not accessible. The system can be characterized by its different vertex types, which can be labeled according to the number of spins pointing toward a vertex. In the square ice, the vertices are named $N_{n}$, where $n$ is the number of spins pointing toward the vertex. Here $N_{0}$ and $N_{4}$ are called double monopoles, $N_{1}$ and $N_{3}$ are monopoles, and $N_{2, \text { biased }}$ and $N_{2, \mathrm{gs}}$ are ice-rule-obeying vertices, where the latter is the ground-state vertex configuration [2]. Similar vertex states appear in the $F$ model [24,25]. In Fig. 1(a) we highlight the different vertex types for particle-based square ice, while Fig. 1(b) shows the same for particle-based hexagonal ice.

When the interactions between neighboring effective spins are weak, the two-dimensional (2D) square ice forms a trivially disordered state containing a finite number of non-ice-rule-obeying vertices. As the interaction strength increases, a second-order phase transition occurs from the disordered state to a long-range-ordered state in which only $N_{2, \mathrm{gs}}$ vertices are present $[2,26]$. The 2D hexagonal ice shows only a crossover from a disordered state with non-ice-ruleobeying vertices to a disordered state in which all vertices obey the ice rule [2]. These assumptions are consistent with behaviors observed in simulations [16,27], theory [28], and 
(a)
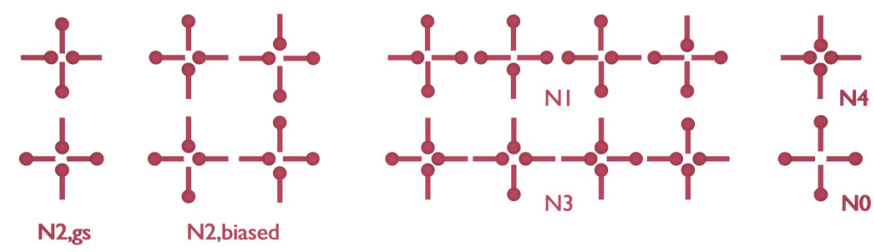

(b)

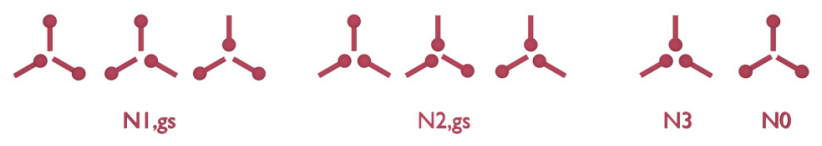

FIG. 1. (a) Vertex types for square ice in a particle-based system. (b) Vertex types for hexagonal ice. Dots indicate the location of the particle with respect to the vertex.

experimental studies of colloidal spin ice $[13,14]$, which show that particle-based and magnetic ASI systems can be mapped to one another.

Previous work on ASI has generally focused on equilibrium states; however, ASI are ideal systems in which to address current issues in nonequilibrium statistical mechanics. For example, the density of topological defects present in a system after it has been quenched at different rates through a second-order phase transition has implications for defect formation in the early universe [29,30], vortex formation at normal to superconducting transitions [31], liquid crystal systems [32], Bose-Einstein condensates [33-35], ion crystals [36,37], and manganites [38]. There are different scenarios describing the behavior of the defect density depending on whether the quench crosses a phase transition and whether the defects diffuse randomly or are strongly interacting. Artificial spin ice systems provide an ideal test bed for exploring these issues since the microscopic degrees of freedom can be directly accessed and there are experimentally achievable ways to move through the different phases in order to induce quench dynamics.

One scenario describing the behavior of the defects for varied quench rates is the Kibble-Zurek (KZ) mechanism $[29,30,39]$, in which the defect density $\rho_{d}$ increases with increasing quench rate according to a universal power law $\rho_{d} \propto$ $\tau_{Q}^{-\beta}$. Here $\tau_{Q}$ is the inverse quench rate describing the time duration of the quench. For large $\tau_{Q}$ or slow quench rates, $\rho_{d}$ is expected to be small. In the $\mathrm{KZ}$ mechanism, $\beta$ is related to the critical exponents associated with the underlying equilibrium second-order phase transition through which the system is quenched. The KZ mechanism relies on the adiabatic-impulse approximation according to which defects are produced when the system falls out of equilibrium, and is thus sensitive to the freeze-out timescale. In addition, it requires that the density of defects arises exclusively from the nonadiabatic crossing of the critical point, and neglects any dynamics in the ordered phase that may alter the defect population. The KZ mechanism is appealing since it can be applied to a wide variety of systems that exhibit phase transitions ranging from cosmological systems to superconductors [39], and it is desirable to understand where the KZ mechanism breaks down or becomes entangled with other possible mechanisms. There is already some numerical work [40] showing that $\mathrm{KZ}$ scaling holds for quenches of the magnetic field in a 3D classical dipolar spin ice with a critical point that falls in the 3D Ising universality class, suggesting that $\mathrm{KZ}$ scaling occurs in at least some classes of spin ice systems. In Ref. [40], the KZ scaling was argued to originate from the slow microscopic timescales of the defect dynamics. It is possible that a $2 \mathrm{D}$ system such as a particle-based ASI would behave differently since monopoles are bound in two dimensions, which could give rise to faster nonadiabatic dynamics [2,14,28].

A competing scenario for the defect density behavior during a quench is a coarsening process facilitated by the motion and annihilation of defects on the ordered side of the phase transition driven by strong defect-defect interactions [41]. When a system obeying this scenario is quenched through a second-order phase transition, the dynamics after the quench could still show a power-law decay of the defect density as a function of quench duration $\tau_{Q}$ if critical coarsening occurs [42]. Here the defects do not move independently during the quench but interact and annihilate, and the defect density reflects this coarsening process instead of the $\mathrm{KZ}$ mechanism.

Artificial spin ice systems are ideal for discriminating between these different scenarios since the ices contain very-well-defined excitations such as monopoles, while the universality class of the phase transition in many types of ASI, including the square ice, is known. Since the square ice exhibits a phase transition but the hexagonal ice does not, these two types of ice should have very different behaviors during a quench. Studies of quench dynamics in ASI systems could also provide insight into the behavior of broader classes of spin ice systems [43-47]. We note that there are two prominent types of ASI system: nanomagnetic and particle based. In our work we focus on the particle-based colloidal ASI system, while we comment on possible differences in behavior of the nanomagnetic ASI in Sec. IV.

In this paper we perform simulations and scaling analysis of a colloidal artificial spin ice. The advantage of colloidal ice is that the strength of the colloid-colloid interactions can be tuned experimentally as a function of time, bringing the system from a noninteracting disordered regime to a strongly interacting ordered regime as a function of changing magnetic field and giving access to a range of different quench rates. We consider both square and hexagonal colloidal ice and start the system in the weakly interacting disordered regime where ice-rule-obeying vertices are not favored. The strength of the repulsive colloidal interactions is increased as a function of time until the system either passes through a second-order phase transition in the case of square ice or crosses into an ice-rule-obeying state in the case of hexagonal ice. In the square ice, the transition into a long-range-ordered state falls into the Ising model universality class. We measure the population of the different vertex types as well as the spatial configurations of the defects for different interaction strength sweep rates. This protocol could be achieved experimentally for paramagnetic colloids by sweeping the external magnetic field.

Our system faithfully mimics the experimental setup described in Refs. [13,14,16,27]. An advantage to studying a particle-based model is that the time-dependent dynamics during the quench can be accessed directly using molecular 
dynamics techniques. This avoids the issues that arise in using Monte Carlo (MC) methods to examine quenched dynamics and $\mathrm{KZ}$ scaling, where it has been shown that different MC methods produce different results [48].

\section{SIMULATION}

We simulate a system of colloidal superparamagnetic particles with a radius of $r=1 \mu \mathrm{m}$. The particles are placed in a square $100 \times 100$ lattice containing 20000 particles and 10000 pinning sites or in a hexagonal $38 \times 66$ lattice containing 15048 particles and 10032 pinning sites. Each pinning site is a double-well trap and the lattice constant is $a_{x}=a_{y}=5.0 \mu \mathrm{m}$ in the square ice lattice and $a_{x}=3 \mu \mathrm{m}$ and $a_{y}=3 \sqrt{3} / 2 \mu \mathrm{m}$ in the hexagonal ice lattice, giving a total system size of $500 \times 500 \mu \mathrm{m}^{2}$ for the square ice and $342 \times 342.95 \mu \mathrm{m}^{2}$ for the hexagonal ice. We use periodic boundary conditions in both the $x$ and $y$ directions.

The elongated gravitational double-well traps are modeled as two spherical quarters connected by an elongated half-cylindrical trough of length $2 \mu \mathrm{m}$ in the square ice and $1.4 \mu \mathrm{m}$ in the hexagonal ice that has a repulsive bump in the middle. Each minimum of the double well is located at the end of the elongated trough, coinciding with the minimum in the spherical quarter. When the particle is in either of the spherical ends, a harmonic spring force tethers the particle to the minimum with a spring constant of $k=0.222 \mathrm{pN} / \mu \mathrm{m}$ for the square ice and $k=2.22 \mathrm{pN} / \mu \mathrm{m}$ for the hexagonal ice. We stay in the experimentally relevant limit where the particles never hop out of the individual double-well traps [13]. When the particle is in the elongated part of the pin, the same harmonic spring force acts on it in the direction perpendicular to the elongated trough, and an additional middle barrier force in the trap is exerted by the bump in the middle of the trough which has a maximum value of $F_{\mathrm{MB}}=0.011$ $\mathrm{pN}$ for the square ice and $F_{\mathrm{MB}}=0.211 \mathrm{pN}$ for the hexagonal ice. This force decays to zero linearly in each half of the elongated trough as the intersection with the spherical quarters is approached. These forces together compose the substrate force denoted by $F_{s}^{i}$.

We use a smaller lattice constant for the less densely packed hexagonal ice because stronger interparticle interactions are required to induce the spin ice ordering compared to the square ice system. We also increase the pinning strength significantly for the hexagonal ice to prevent the particles from ordering into a triangular lattice with each particle sitting at the center of the elongated trough, which destroys the spin ice nature of the particle based system. With the chosen values, which are within the experimentally realizable regime, the spin ice manifold is preserved.

Magnetization of the particles in the $z$ direction by an external magnetic field produces a repulsive particleparticle interaction force $F_{\mathrm{pp}}(r)=A_{\mathrm{c}} / r^{4}$, with $A_{\mathrm{c}}=3 \times$ $10^{6} \chi_{m}^{2} V^{2} 2 B^{2} / 2 \pi \mu$ for particles a distance $r$ apart $[14,27]$. Here $\chi_{m}$ is the magnetic susceptibility, $V$ is the particle volume, $B$ is the magnetic field in millitesla, and all distances are measured in $\mu$. The interaction falls off as $1 / r^{3}$, which is rapid enough that it can be cut off beyond three lattice constants. We have found that larger cutoffs produce essentially the same results [27]. At $B=40 \mathrm{mT}$, the maximum field we consider, this gives $F_{\mathrm{pp}}=0.49 \mathrm{pN}$ for $r=3 \mu \mathrm{m}$, which is a typical distance for the square ice, and $F_{\mathrm{pp}}=6.05 \mathrm{pN}$ for $r=1.6 \mu \mathrm{m}$, which is a typical distance for the hexagonal ice. All of these parameters are chosen to match the length and timescales of an experimental magnetic spin ice system [13,14,27].

The dynamics of colloid $i$ are obtained using the discretized overdamped equation of motion

$$
\frac{1}{\mu} \frac{\Delta \mathbf{r}_{i}}{\Delta t}=\sqrt{\frac{2}{D \Delta t}} k_{\mathrm{B}} T N[0,1]+F_{\mathrm{pp}}^{i}+F_{\mathrm{s}}^{i} .
$$

Here the diffusion constant $D=36000 \mathrm{~nm}^{2} / \mathrm{s}$, the mobility $\mu=8.894 \mu \mathrm{m} / \mathrm{s} / \mathrm{pN}$, and the simulation time step $\Delta t=1$ $\mathrm{ms}$ [27]. The first term on the right-hand side is a thermal force consisting of Langevin kicks of magnitude $F_{T}=0.954$ $\mathrm{pN}$ corresponding to a temperature of $T=20^{\circ} \mathrm{C}[16,27]$. Here $N[0,1]$ denotes a random number drawn from a normal (Gaussian) distribution with a mean of 0 and a standard deviation of 1 . Each trap is filled with a single particle which is randomly placed in one of the two minima. We increase $B$ linearly from $B=0 \mathrm{mT}$ to $B=40 \mathrm{mT}$, following a procedure that is feasible to achieve experimentally. We average the results over 100 simulations performed with different random seeds.

In Fig. 2(a) we illustrate the colloid positions in a representative square ice vertex state, where arrows indicate the net colloid-colloid interaction forces $F_{\mathrm{pp}}^{i}$ on colloid $i$. Each colloid sits in one of the two minima found within its confining trap. For the colloid in the upper trap, $F_{\mathrm{pp}}^{i}$ is parallel to the axis of the confining trap and points away from the vertex center, while for the colloids in the horizontal traps, $F_{\mathrm{pp}}^{i}$ is at an angle to the axis of the confining trap. The magnitude of $F_{\mathrm{pp}}^{i}$ increases with increasing magnetic field $B$. In the inset of Fig. 2(b) we plot $F_{\mathrm{pp}}^{i}$ versus colloid-colloid spacing $r$, showing that the interaction force decreases as $r$ increases but increases as $B$ becomes larger. The main panel of Fig. 2(b) shows the magnitude of $F_{\mathrm{pp}}^{i}$ at a specific value of $r$ as a function of $B$, showing that $F_{\mathrm{pp}}^{i}=0$ when $B=0$ and that $F_{\mathrm{pp}}^{i}$ increases with increasing $B$. When $B=0$, the force arrows in Fig. 2(a) disappear and the ice-rule-obeying vertex states are no longer energetically favorable, so the vertex types are randomly populated. We hold the temperature of our system fixed in order to model the experimentally relevant situation in which the relative strength of $B^{2} / T$ is changed only by varying $B$. If $B$ is slowly increased adiabatically, there is a critical second-order phase transition in the square ice at which $F_{\mathrm{pp}}^{i}$ overwhelms the thermal fluctuations and the system enters the long-range-ordered square ice-rule-obeying state in which all of the vertices are in the $N_{2, \mathrm{gs}}$ configuration. In the hexagonal ice, there is no symmetry breaking in the ice-rule-obeying state, so there is only a crossover but no phase transition as the system shifts from a disordered state in which the vertices are populated randomly to a disordered state in which all of the vertices obey the ice rule.

\section{RESULTS}

In Fig. 3(a) we show a schematic of the magnetically interacting colloids in a square ASI of double-well traps and in Fig. 3(b) we show the corresponding hexagonal ASI. Each 

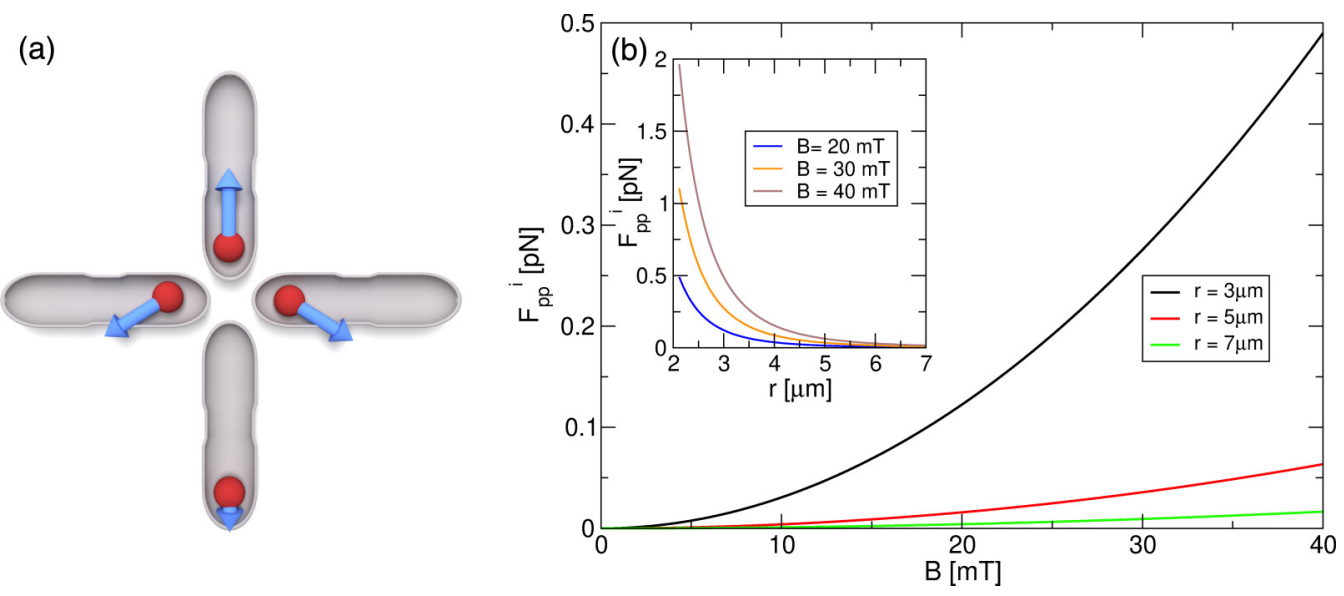

FIG. 2. (a) Schematic illustrating the forces (blue arrows) experienced by the colloids (red circles) in the double-well traps (gray) surrounding an individual vertex in a square ice system. Each colloid sits at one end of its confining double-well trap. (b) Colloid-colloid interaction force $F_{\mathrm{pp}}^{i}$ vs applied magnetic field strength $B$ at three colloid-colloid spacings of $r=3$ (black), 5 (red), and $7 \mu \mathrm{m}$ (green). The inset shows $F_{\mathrm{pp}}^{i}$ vs $r$ at three magnetic field strengths of $B=20$ (blue), 30 (orange), and $40 \mathrm{mT}$ (brown).

elongated trap holds a single colloid which can sit on either end of the trap, determining the direction of the effective spin. For our parameters, the critical magnetic field at which the equilibrium system orders into a defect-free ground state is $B_{c}=9 \mathrm{mT}$. We start the system at $B=0$ and increase the field to $B=40 \mathrm{mT}$ in a time of $\tau_{Q}$, given in seconds. The fastest value of $\tau_{Q}$ we consider is $10 \mathrm{~s}$, while the slowest is $\tau_{Q}=$ $6000 \mathrm{~s}$, which is close to the adiabatic limit. These timescales are experimentally accessible. Figure 4 shows the vertex populations with the same color scheme from Fig. 3(a) in a simulation with a quench time duration of $\tau_{Q}=80 \mathrm{~s}$ at several values of $B$ corresponding to several different instants of time

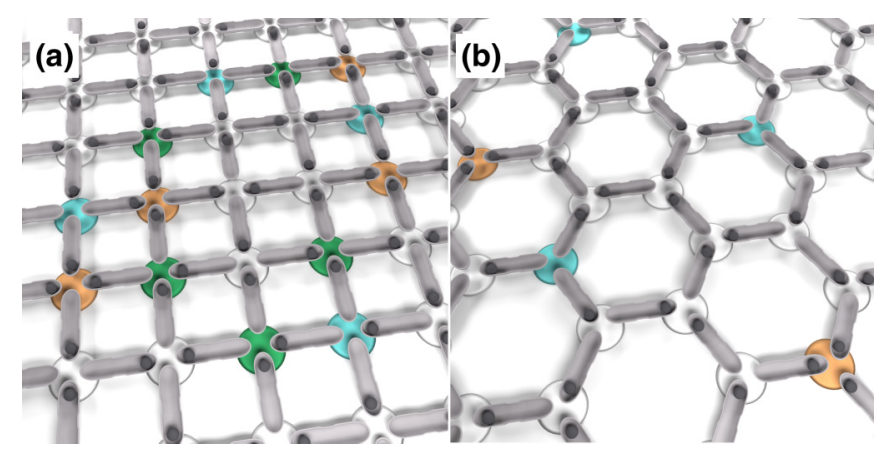

FIG. 3. (a) Schematic of the square ice system. Lozenges are the double-well traps which are each occupied by a single colloid, shown as a gray sphere, that preferentially sits at one of the two ends of the trap. In experimental realizations, the colloidal particles are paramagnetic and repel each other with a strength that can be controlled using an applied magnetic field. The circles underneath each vertex are colored according to the vertex type: $N_{2, \mathrm{gs}}$ (the ground state), white; $N_{2, \text { biased }}$, green; $N_{1}$, light blue; and $N_{3}$, orange. Here there are no highly unfavorable $N_{0}$ or $N_{4}$ vertices. At the center of the image is a ground-state cluster of vertices surrounded by a grain boundary which separates it from vertices in a ground state with the opposite orientation. (b) Schematic of the hexagonal ice system. The circles underneath each vertex are colored according to the vertex type: $N_{1}$ and $N_{2}$ (the ice rule obeying states), white; $N_{3}$, orange; and $N_{0}$, blue. $t$. The defects form closed loop grain boundaries similar to those observed in square ice systems with varied amounts of quenched disorder $[10,17,49,50]$. For faster quench rates corresponding to smaller $\tau_{Q}$, the number of non-ground-state vertices increases and the grain boundaries are smaller.

In Fig. 5(a) we plot the fraction of non-ground-state vertices $N_{\text {ngs }} / N$ versus $B$ at different sweep rates. Note that the value of $B$ is changing as a function of time, so it is possible to

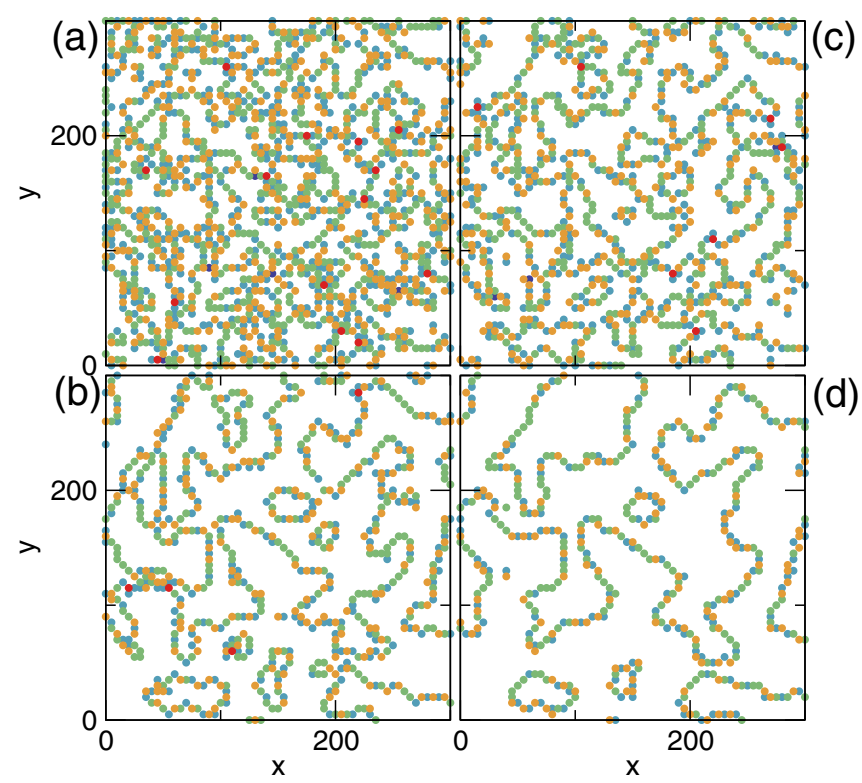

FIG. 4. Coarsening in the square ice system shown as snapshots of a small portion of the system with quench time $\tau_{Q}=80 \mathrm{~s}$. Each snapshot shows a different value of the magnetic field, which is being swept dynamically from $B=0 \mathrm{mT}$ to $B=40 \mathrm{mT}$ : (a) $B=16 \mathrm{mT}$ at a time of $t=32 \mathrm{~s}$, (b) $B=20 \mathrm{mT}$ at a time of $t=40 \mathrm{~s}$, (c) $B=24 \mathrm{mT}$ at a time of $t=48 \mathrm{~s}$, and (d) $B=30 \mathrm{mT}$ at a time of $t=60 \mathrm{~s}$. Dark blue and red dots show $N_{0}$ and $N_{4}$ vertices (double monopoles), blue and orange dots $N_{1}$ and $N_{3}$ vertices (monopoles), and green

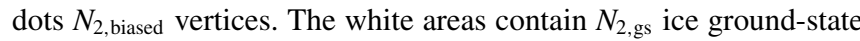
vertices. 

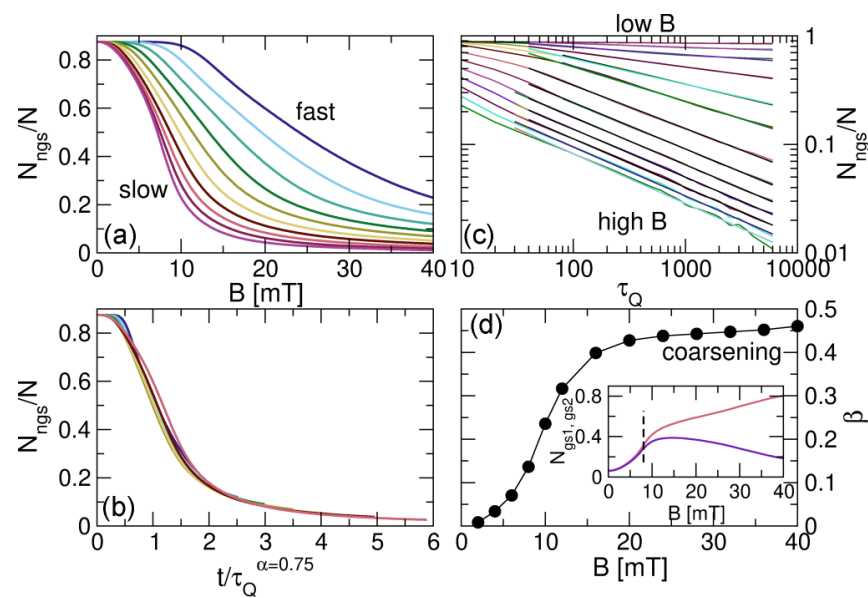

FIG. 5. Transition from the ordered to the disordered state as a function of quenching speed in the square ice system. (a) Fraction of non-ground-state vertex types $N_{\text {ngs }} / N$ in the system vs magnetic field $B$. From dark blue to dark red, the curves represent quench times of $\tau_{Q}=10,20,40,80,150,300,600,1200,2500$, and $6000 \mathrm{~s}$. (b) Rescaling of the data in (a) plotted as $N_{\text {ngs }} / N$ vs $t / \tau_{Q}^{\alpha}$, the elapsed time $t$ divided by the total quench time raised to the power $\alpha=0.75$. (c) Scaling of $N_{\text {ngs }} / N$ as a function of quench time $\tau_{Q}$ for different magnetic field values. From dark blue to dark red, the curves represent constant magnetic fields of $B=4 \mathrm{mT}$ (dark blue), $B=6 \mathrm{mT}$ (light blue), $B=8 \mathrm{mT}$ (light green), and subsequently $B=10,12$, 16, 20, 24, 28, 32, 36, and $40 \mathrm{mT}$. (d) Power-law exponents $\beta$ obtained from the data in (c) vs the magnetic field value $B$. The inset shows the fraction of the larger ground-state cluster $n_{\mathrm{gs} 1}=N_{\mathrm{gs} 1} / N$ (upper pink line) and the fraction of the smaller ground-state cluster $n_{\mathrm{gs} 2}=N_{\mathrm{gs} 2} / N$ (lower purple line) as a function of $B$, showing a bifurcation at the critical field (dashed line), corresponding to the spontaneous symmetry breaking.

rescale the $x$ axis and plot the curves as a function of elapsed time $t$. The fastest transition with $\tau_{Q}=10 \mathrm{~s}$ is denoted by the rightmost blue line and the quench rate decreases for curves that are further to the left. The systems are initialized in a completely random configuration at $B=0$ with $N_{\text {ngs }} / N=7 / 8$. As the quench rate decreases, the value of $N_{\text {ngs }} / N$ decreases. In Fig. 5(b) we show that the $N_{\text {ngs }} / N$ curves from Fig. 5(a) can be replotted as a function of time $t$ and then collapsed by dividing the time $t$ by $\tau_{Q}^{\alpha}$, where $\alpha=3 / 4$.

In Fig. 5(c) we plot $N_{\text {ngs }} / N$ versus the quench time $\tau_{Q}$ at different fixed values of the magnetic field from $B=4 \mathrm{mT}$ (top) to $B=40 \mathrm{mT}$ (bottom). The runs were performed over the range of total time $\tau_{Q}=10 \mathrm{~s}$ to $\tau_{Q}=6000 \mathrm{~s}$, which is an experimentally accessible range of timescales for changing magnetic fields $[13,14,27]$. We fit each curve to a power law with

$$
N_{\text {ngs }} / N \propto \tau_{Q}^{-\beta},
$$

where $N_{\text {ngs }} / N=\rho_{d}$, and we plot the resulting exponents $\beta$ versus $B$ in Fig. 5(d). For $B<9 \mathrm{mT}$, the system does not order at all, while for $B>12 \mathrm{mT}$, the exponent saturates at $\beta=0.45$. This indicates that we have two different regimes of behavior. For smaller magnetic fields between the values of $B=10 \mathrm{mT}$ and $B=12 \mathrm{mT}$, we find a slower decay rate with an exponent between $\beta=0.2$ and $\beta=0.3$.

\section{A. Kibble-Zurek mechanism}

Now that we have established that our system has both a critical point and power-law scaling of the defect density for different quench rates, we can test whether our results are consistent with the KZ mechanism [29,30,39]. In particular, the lag time between the nonequilibrium and equilibrium value scales is expected to be set by the so-called freeze-out time

$$
\hat{t} \sim \tau_{Q}^{z v /(1+z v)},
$$

where $z$ is the dynamic exponent and $v$ is the correlation length exponent [39].

To investigate whether the transition obeys the KZ mechanism, we collapse the runs with different quench times together by rescaling the time axis. In Fig. 5(b) we show the evolution of $N_{\text {ngs }} / N$ versus time where the time has been divided by a power of the quench time $\tau_{Q}^{\alpha}$. The collapse is achieved by setting $\alpha=3 / 4$. The $\mathrm{KZ}$ mechanism prediction then implies that $\frac{z v}{1+z v}=\alpha=3 / 4$; however, the square ice falls into the Ising universality class, which has $v=1$ and $z=$ 2 [51], as was also established experimentally [26]. Inserting the numbers expected for the Ising model gives $\frac{z v}{1+z v}=2 / 3$, which is different from the value $3 / 4$ that we find. This implies that either our system obeys $\mathrm{KZ}$ scaling in a universality class different from the 2D Ising model or that the power law we observe should be attributed to the alternative mechanism of critical coarsening.

Another prediction of the $\mathrm{KZ}$ mechanism is that the total number of defects should scale as

$$
\rho_{d} \sim \tau_{Q}^{-D v /(1+z v)},
$$

where $D$ is the dimension of the system. In our case, $D=2$ and $\rho_{d}=N_{\text {ngs }} / N$. The 2D Ising model gives a prediction of $\frac{D v}{1+z v}=2 / 3$, but in Fig. 5(c) we find $\rho_{d} \sim \tau_{Q}^{-1 / 2}$ or $\frac{D v}{1+z v}=$ $1 / 2$, indicating that the scaling of the defects that we obtain does not agree with the $\mathrm{KZ}$ mechanism in the 2D Ising universality class. This could be due to the strong interactions between the defects, since for quenches out to higher values of $B$, the defects such as +1 and -1 monopoles are strongly interacting and undergo a non-negligible amount of motion during the quench via their effective Coulomb interactions, as has been observed in colloidal experiments [14] and simulations [27].

The presence of defect dynamics during the part of the quench that approaches the ordered state violates one of the assumptions for the validity of the $\mathrm{KZ}$ scenario. We note that for coarsening dynamics near a critical point, ordered regions of radius $R$ grow as $R(t) \propto t^{1 / z}$ [42], which for the Ising model gives

$$
R(t) \propto t^{1 / 2}
$$

where $t$ is time. In our case, the onset of coarsening begins once $B$ is large enough that the equilibrium system would be in the longer-range-ordered phase. If the size of the ordered regions grows, the number of defects would be proportional to $1 / R(t)$, in agreement with our observations. We also note that direct observation of the dynamics through the quench reveals behavior consistent with defect-defect interactions, such as defects aggregating by moving in straight lines toward each 


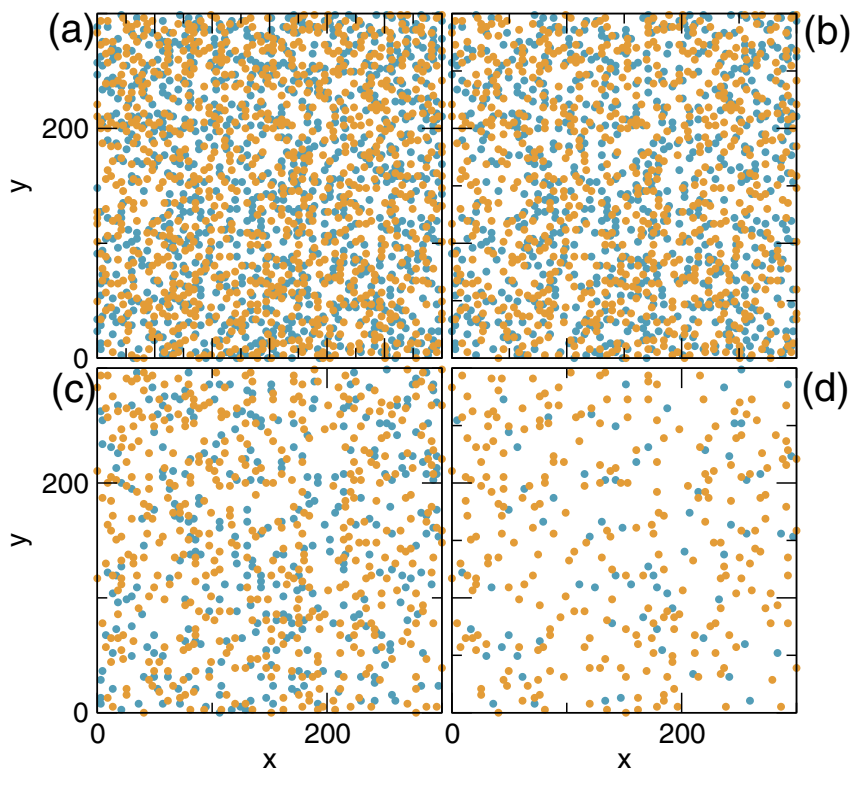

FIG. 6. Transition in the hexagonal ASI shown as snapshots of a small part of the system with quench time $\tau_{Q}=80 \mathrm{~s}$. Each snapshot shows a different value of the magnetic field, which is being swept dynamically from $B=0 \mathrm{mT}$ to $B=40 \mathrm{mT}$ : (a) $B=8 \mathrm{mT}$ at a time of $t=16 \mathrm{~s}$, (b) $B=10 \mathrm{mT}$ at a time of $t=20 \mathrm{~s}$, (c) $B=12 \mathrm{mT}$ at a time of $t=24 \mathrm{~s}$, and (d) $B=14 \mathrm{mT}$ at a time of $t=28 \mathrm{~s}$. Blue dots show three-out vertices and orange dots three-in vertices. White regions contain ground-state vertices that are not plotted.

other, suggesting that there is a strong Coulomb interaction between the defects.

In other types of ASI, such as nanomagnetic systems, it is possible that the $\mathrm{KZ}$ regime could be accessed more easily since the motion of the defects may be slower due to the presence of quenched disorder in the nanomagnetic islands. Alternatively, at much faster quench rates than those we consider, there could be a regime of $\mathrm{KZ}$ behavior in which the defects simply do not have time to move. There is some recent Monte Carlo-based numerical work on magnetic spin ice systems in which the quench dynamics was shown to be consistent with the KZ mechanism [52]. For 3D magnetic spin ice systems there is evidence for KZ scaling [40]; however, in three dimensions, defects such as monopoles are not bound and are able to wander freely. It was argued in Ref. [40] that the slow dynamics of such wandering defects makes it possible to access the $\mathrm{KZ}$ regime.

\section{B. Hexagonal system}

In the hexagonal colloidal ice, each vertex is surrounded by only three elongated pinning sites. The colloidal hexagonal ice shows a crossover from a disordered paramagnetic state at low $B$ with three-in and three-out monopoles to a disordered ice-rule-obeying state with two-in/one-out and two-out/onein vertices at higher $B$ [13]. The lack of long-range order in the ice rule state means that there is no phase transition between the paramagnetic and ice-rule-obeying states. As a result, we would not expect the $\mathrm{KZ}$ mechanism to apply to the hexagonal colloidal ice, nor do we expect critical coarsening to occur. We conduct the same type of simulation described above in
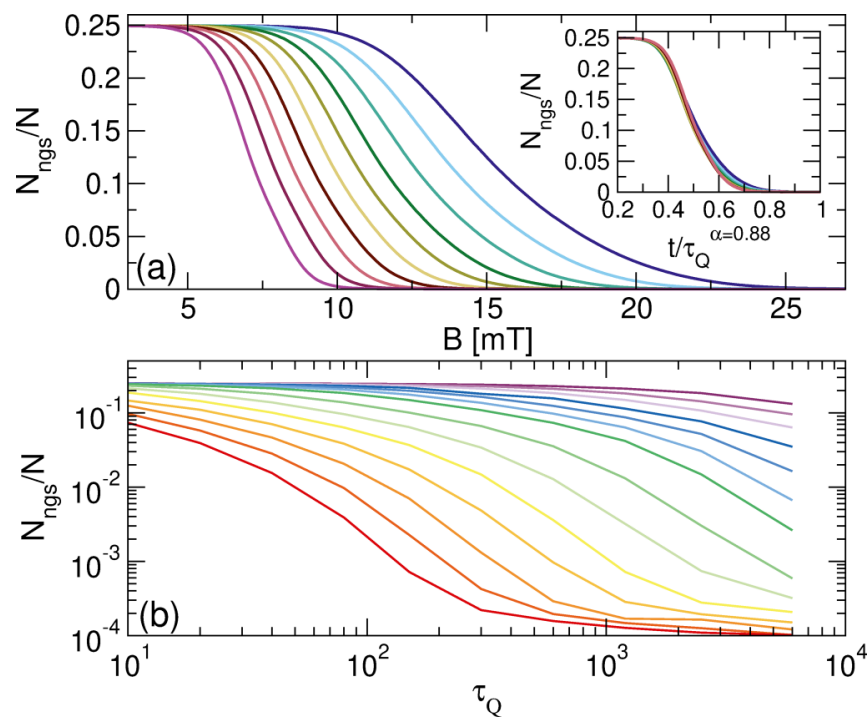

FIG. 7. (a) Measure of the transition in the hexagonal ASI system. Non-ground-state vertex fraction $N_{\text {ngs }} / N$ as a function of applied field $B$ for $\tau_{Q}=10,20,40,80,150,300,600,1200,2500$, and $6000 \mathrm{~s}$, from blue (right) to red (left). The inset shows rescaling of $N_{\text {ngs }} / N$ as a function of elapsed time $t$ divided by $\tau_{Q}^{\alpha}$, where $\alpha=0.88$. (b) A log-log plot of $N_{\text {ngs }} / N$ vs $\tau_{Q}$ for the system in (a) at $B=7.0$, $7.5,8,8.5,9,9.5,10,11,12,13,14,15,16$, and $17 \mathrm{mT}$, from purple (top) to red (bottom), showing the lack of a power-law decay of defects, in contrast to the square ice case.

which we pass from a zero-field state to a maximum field of $B=40 \mathrm{mT}$ during a quench time $\tau_{Q}$. Here the equilibrium configurations at higher $B$ would not contain any monopoles. In Fig. 6 we show snapshots of the transition in the hexagonal ice as a function of increasing interaction strength $B=8$, 10,12 , and $14 \mathrm{mT}$ corresponding to consecutive times of $16,20,24$, and $28 \mathrm{~s}$ for a system with a quench time of $\tau_{Q}=80 \mathrm{~s}$. In this case, nonfavorable vertex types disappear during the crossover to the disordered ice-rule-obeying state without forming any spatially correlated structures or grain boundaries of the type observed in the square ice system. Therefore, we expect the defect dynamics and coarsening to be different in the hexagonal and square ices. In the initial random configuration, the ground-state vertices in the hexagonal ice already occupy $N_{\mathrm{gs}} / N=3 / 4$ of the system. This is in contrast to the square ice system, where $N_{\mathrm{gs}} / N=1 / 8$ at initialization. As a result, it is not necessary for the hexagonal ice to nucleate and grow clusters of ice-rule-obeying vertices. Additionally, power-law critical coarsening does not occur in the hexagonal ice since there is no underlying phase transition.

In Fig. 7 we plot the number of non-ground-state vertices as a function of the applied field for different $\tau_{Q}$ values ranging from $\tau_{Q}=10 \mathrm{~s}$ to $\tau_{Q}=6000 \mathrm{~s}$ for the hexagonal ice. Here the crossover to an ice-rule-obeying state happens over a narrower range of $B$ than in the square ice since there are no kinetic barriers to overcome in the process of eliminating the non-ice-rule-obeying defects. This produces a higher exponent of $\alpha=0.88$ when we create a scaling plot of $N_{\text {ngs }} / N$ versus $t / \tau_{Q}^{\alpha}$ as shown in the inset of Fig. 7, indicating that the defect annihilation mechanism differs from what is found in the square ice system. In the square ice, the 
monopoles are located along grain boundaries and annihilate as the grain boundaries shrink. In contrast, the monopoles in the hexagonal ice are not on grain boundaries and can move toward each other along straight paths, as observed experimentally [8]. More relevantly, monopoles in hexagonal ice are not topologically protected. While charges \pm 2 of square ice cannot be reabsorbed but can only be annihilated and created in pairs, \pm 3 charged violations of the ice rule in hexagonal ice can appear and disappear individually. This is because in the hexagonal ice, even the ice-rule-obeying vertices are charged $( \pm 1)$, and thus each monopole in the hexagonal ice can transfer charge to the surrounding plasma.

In Fig. 7(b), the plot of defect density $N_{\text {ngs }} / N$ versus the quench duration $\tau_{Q}$ for the hexagonal ice at varied $B$ shows that there is no power-law behavior in the density of defects, which is consistent with the lack of a phase transition in the system. As a result, the $\mathrm{KZ}$ mechanism scenario does not apply, and critical coarsening cannot occur due to the lack of a critical point.

\section{DISCUSSION}

Our results can be compared directly to experiments on colloidal ice. Extending the results to the case of magnetic ice requires more caution. Up to a nearest-neighbor approximation, a magnetic square ice can be mapped into a $J_{1}-J_{2}$ Ising system [53]. The colloidal square ice differs from the magnetic square ice in both its energetics and the nature of its frustration [16,54]. An equilibrium colloidal square ice can be mapped into a magnetic square ice as shown in Refs. [26,28,55], but the equilibrium phase transition has a different nature in the magnetic and colloidal spin ices. As described in Ref. [27], the magnetic spin ice system minimizes the energy of each local vertex, whereas the colloidal spin ice system minimizes the global Coulomb energy. Additionally, the colloidal ice contains many more states, corresponding to colloids located in between preferential positions, which can make its out-of-equilibrium kinetics much different from those of its magnetic analog. As a result, although the colloidal ice and magnetic ice have similar equilibrium phases [28], the dynamics could be different in the two systems.

It is possible that the coarsening dynamics in the magnetic square ASI could differ from that found in the square particle-based ASI since the particle-based system minimizes the global energy rather than the vertex energy, making the resulting ice state more fragile [16,28]. It has also been demonstrated that the motion of defects in colloid ice differs from the motion in magnetic ice since +1 and +3 monopoles move at different speeds in the colloidal ice due to their differing energies [27], but move at equal speeds in the magnetic ice since their energies in the magnetic system are equal. The kinetics of annihilation and spin flipping in magnetic ice are also likely to depend on the microscopic details of the particular magnetic ASI realization.

It should be possible to perform quenches across transitions or crossovers in magnetic ASI by sweeping temperature in order to pass from a higher-temperature liquid to a lower-temperature ordered state [2,56,57]. Quenches can be performed by varying the rate at which the temperature is swept across the transition or crossover. In colloidal systems, beyond changing the magnetic field it would also be possible to consider temperature-induced quenches, although the fastest accessible quench time would be longer compared to the magnetic quenches. In superconducting systems, where particle-based artificial ices can be realized using magnetic flux lines in specially designed pinning sites, a similar temperature control could be used at finite fields to pass from a normal to a superconducting state as a function of temperature.

In our system, it is likely that the strong interactions between the defects result in coarsening behavior. Therefore, it may be possible to access a regime in which the $\mathrm{KZ}$ mechanism would be valid by reducing the defect-defect interaction strength. One possible experiment to test this would be to create magnetic nanoislands that are sufficiently far apart to reduce the strength of the defect-defect interactions, which could minimize the coarsening. Other future directions are to consider alternative ASI geometries [2,11,16,58-60], including geometries in which the monopoles are not as strongly bound [61,62]. It would also be interesting to study the effect of disorder to see whether the exponents change or if glassy dynamics arise, which could produce a crossover to a logarithmic behavior rather than a power-law decay. It may also be possible that a small amount of disorder could slow down the dynamics of the defects enough to make the $\mathrm{KZ}$ mechanism accessible.

\section{CONCLUSION}

In conclusion, we have examined the defect density populations for varied quench rates from a disordered to an ordered state in square and hexagonal colloidal spin ice systems, where a magnetic field is used to tune the strength of the interactions between the effective spins. In the square ice, we find that when the quench into the ordered state is sufficiently deep, there is a power-law decay of the defect density with $\rho_{d} \propto \tau_{Q}^{-1 / 2}$. Based on scaling arguments for the universality class of the square ice, we find that the behavior of the quenched square ice is most likely governed by critical coarsening rather than the Kibble-Zurek mechanism. The lack of $\mathrm{KZ}$ behavior could be due to the strong Coulomb interactions between the monopoles, which arise because particle-based spin ice models minimize the global Coulomb energy rather than the local vertex energy. These stronger interactions permit the defects to move toward each other nondiffusively, whereas the $\mathrm{KZ}$ mechanism assumes that no dynamics occurs in the ordered phase. It is also possible that the colloidal ice falls into a universality class different from the 2D Ising model observed for magnetic spin ice, and therefore it is not possible to rule out the $\mathrm{KZ}$ mechanism for the colloidal square ice. In the case of the colloidal hexagonal ice, which has no secondorder phase transition to an ordered state, we find distinctive defect configurations, no grain boundaries, and no power-law scaling of the defect density under varied quench rates. Our results could be compared with quenches of different types of ASI in magnetic, colloidal, and superconducting systems. Each of these systems could exhibit different interactions between the defects or different kinetics and it is possible that one or more of the systems could have a regime in which the $\mathrm{KZ}$ mechanism is observable. 


\section{ACKNOWLEDGMENTS}

We gratefully acknowledge the support of the U.S. Department of Energy through the LANL/LDRD program for this work. This work was supported by the U.S. Department of Energy through the Los Alamos National Laboratory. Los Alamos National Laboratory is operated by Triad National Security, LLC, for the National Nuclear Security Administration of the U.S. Department of Energy (Contract No. 892333218NCA000001).
[1] R. F. Wang, C. Nisoli, R. S. Freitas, J. Li, W. McConville, B. J. Cooley, M. S. Lund, N. Samarth, C. Leighton, V. H. Crespi, and P. Schiffer, Artificial 'spin ice' in a geometrically frustrated lattice of nanoscale ferromagnetic islands, Nature (London) 439, 303 (2006).

[2] C. Nisoli, R. Moessner, and P. Schiffer, Colloquium: Artificial spin ice: Designing and imaging magnetic frustration, Rev. Mod. Phys. 85, 1473 (2013).

[3] I. Gilbert, C. Nisoli, and P. Schiffer, Frustration by design, Phys. Today 69 (7), 54 (2016).

[4] V. Schanilec, Y. Perrin, S. Le Denmat, B. Canals, and N. Rougemaille, Artificial vertex systems by design, arXiv:1902.00452.

[5] S. H. Skjaervø, C. H. Marrows, R. L. Stamps, and L. J. Heyderman, Advances in artificial spin ice, Nat. Rev. Phys. 2, 13 (2019).

[6] Y. Qi, T. Brintlinger, and J. Cumings, Direct observation of the ice rule in an artificial kagome spin ice, Phys. Rev. B 77, 094418 (2008).

[7] S. Ladak, D. E. Read, G. K. Perkins, L. F. Cohen, and W. R. Branford, Direct observation of magnetic monopole defects in an artificial spin-ice system, Nat. Phys. 6, 359 (2010).

[8] E. Mengotti, L. J. Heyderman, A. F. Rodríguez, F. Nolting, R. V. Hügli, and H.-B. Braun, Real-space observation of emergent magnetic monopoles and associated Dirac strings in artificial kagome spin ice, Nat. Phys. 7, 68 (2011).

[9] S. Zhang, I. Gilbert, C. Nisoli, G.-W. Chern, M. J. Erickson, L. O'Brien, C. Leighton, P. E. Lammert, V. H. Crespi, and P. Schiffer, Crystallites of magnetic charges in artificial spin ice, Nature (London) 500, 553 (2013).

[10] J. P. Morgan, A. Stein, S. Langridge, and C. H. Marrows, Thermal ground-state ordering and elementary excitations in artificial magnetic square ice, Nat. Phys. 7, 75 (2011).

[11] Y.-L. Wang, Z.-L. Xiao, A. Snezhko, J. Xu, L. E. Ocola, R. Divan, J. E. Pearson, G. W. Crabtree, and W.-K. Kwok, Rewritable artificial magnetic charge ice, Science 352, 962 (2016).

[12] A. Libál, C. Reichhardt, and C. J. O. Reichhardt, Realizing Colloidal Artificial Ice on Arrays of Optical Traps, Phys. Rev. Lett. 97, 228302 (2006).

[13] A. Ortiz-Ambriz and P. Tierno, Engineering of frustration in colloidal artificial ices realized on microfeatured grooved lattices, Nat. Commun. 7, 10575 (2016).

[14] J. Loehr, A. Ortiz-Ambriz, and P. Tierno, Defect Dynamics in Artificial Colloidal Ice: Real-Time Observation, Manipulation, and Logic Gate, Phys. Rev. Lett. 117, 168001 (2016).

[15] D. Y. Lee and P. Tierno, Energetics and the ground state quest in an artificial triangular colloidal ice, Phys. Rev. Mater. 2, 112601 (2018).
[16] A. Libál, D. Y. Lee, A. Ortiz-Ambriz, C. Reichhardt, C. J. O. Reichhardt, P. Tierno, and C. Nisoli, Ice rule fragility via topological charge transfer in artificial colloidal ice, Nat. Commun. 9, 4146 (2018).

[17] A. Libál, C. J. O. Reichhardt, and C. Reichhardt, Creating Artificial Ice States using Vortices in Nanostructured Superconductors, Phys. Rev. Lett. 102, 237004 (2009).

[18] M. L. Latimer, G. R. Berdiyorov, Z. L. Xiao, F. M. Peeters, and W. K. Kwok, Realization of Artificial Ice Systems for Magnetic Vortices in a Superconducting MoGe Thin Film with Patterned Nanostructures, Phys. Rev. Lett. 111, 067001 (2013).

[19] J. Trastoy, M. Malnou, C. Ulysse, R. Bernard, N. Bergeal, G. Faini, J. Lesueur, J. Briatico, and J. E. Villegas, Freezing and thawing of artificial ice by thermal switching of geometric frustration in magnetic flux lattices, Nat. Nanotechnol. 9, 710 (2014).

[20] C. Xue, J.-Y. Ge, A. He, V. S. Zharinov, V. V. Moshchalkov, Y. H. Zhou, A. V. Silhanek, and J. Van de Vondel, Mapping degenerate vortex states in a kagome lattice of elongated antidots via scanning Hall probe microscopy, Phys. Rev. B 96, 024510 (2017).

[21] Y.-L. Wang, X. Ma, J. Xu, Z.-L. Xiao, A. Snezhko, R. Divan, L. E. Ocola, J. E. Pearson, B. Janko, and W.-K. Kwok, Switchable geometric frustration in an artificial-spinice-superconductor heterosystem, Nat. Nanotechnol. 13, 560 (2018).

[22] G. Möller and R. Moessner, Artificial Square Ice and Related Dipolar Nanoarrays, Phys. Rev. Lett. 96, 237202 (2006).

[23] A. Libál, C. Nisoli, C. J. O. Reichhardt, and C. Reichhardt, Inner Phases of Colloidal Hexagonal Spin Ice, Phys. Rev. Lett. 120, 027204 (2018).

[24] E. H. Lieb, Exact Solution of the $F$ Model of an Antiferroelectric, Phys. Rev. Lett. 18, 1046 (1967).

[25] F. Rys, Über ein zweidimensionales klassisches konfigurationsmodell, Ph.D. thesis, ETH Zürich, 1963.

[26] O. Sendetskyi, V. Scagnoli, N. Leo, L. Anghinolfi, A. Alberca, J. Lüning, U. Staub, P. M. Derlet, and L. J. Heyderman, Continuous magnetic phase transition in artificial square ice, Phys. Rev. B 99, 214430 (2019).

[27] A. Libál, C. Nisoli, C. Reichhardt, and C. J. O. Reichhardt, Dynamic control of topological defects in artificial colloidal ice, Sci. Rep. 7, 651 (2017).

[28] C. Nisoli, Unexpected Phenomenology in Particle-Based Ice Absent in Magnetic Spin Ice, Phys. Rev. Lett. 120, 167205 (2018).

[29] T. W. B. Kibble, Topology of cosmic domains and strings, J. Phys. A: Math. Gen. 9, 1387 (1976).

[30] W. H. Zurek, Cosmological experiments in superfluid helium? Nature (London) 317, 505 (1985). 
[31] R. Monaco, J. Mygind, R. J. Rivers, and V. P. Koshelets, Spontaneous fluxoid formation in superconducting loops, Phys. Rev. B 80, 180501(R) (2009).

[32] M. J. Bowick, L. Chandar, E. A. Schiff, and A. M. Srivastava, The cosmological Kibble mechanism in the laboratory: String formation in liquid crystals, Science 263, 943 (1994).

[33] C. N. Weiler, T. W. Neely, D. R. Scherer, A. S. Bradley, M. J. Davis, and B. P. Anderson, Spontaneous vortices in the formation of Bose-Einstein condensates, Nature (London) 455, 948 (2008).

[34] G. Lamporesi, S. Donadello, S. Serafini, F. Dalfovo, and G. Ferrari, Spontaneous creation of Kibble-Zurek solitons in a Bose-Einstein condensate, Nat. Phys. 9, 656 (2013).

[35] N. Navon, A. L. Gaunt, R. P. Smith, and Z. Hadzibabic, Critical dynamics of spontaneous symmetry breaking in a homogeneous Bose gas, Science 347, 167 (2015).

[36] S. Ulm, J. Roßnagel, G. Jacob, C. Degüenther, S. T. Dawkins, U. G. Poschinger, R. Nigmatullin, A. Retzker, M. B. Plenio, F. Schmidt-Kaler, and K. Singer, Observation of the Kibble-Zurek scaling law for defect formation in ion crystals, Nat. Commun. 4, 2290 (2013).

[37] K. Pyka, J. Keller, H. L. Partner, R. Nigmatullin, T. Burgermeister, D. M. Meier, K. Kuhlmann, A. Retzker, M. B. Plenio, W. H. Zurek, A. del Campo, and T. E. Mehlstäubler, Topological defect formation and spontaneous symmetry breaking in ion Coulomb crystals, Nat. Commun. 4, 2291 (2013).

[38] S. M. Griffin, M. Lilienblum, K. T. Delaney, Y. Kumagai, M. Fiebig, and N. A. Spaldin, Scaling Behavior and Beyond Equilibrium in the Hexagonal Manganites, Phys. Rev. X 2, 041022 (2012).

[39] A. del Campo and W. H. Zurek, Universality of phase transition dynamics: Topological defects from symmetry breaking, Int. J. Mod. Phys. A 29, 1430018 (2014).

[40] J. Hamp, A. Chandran, R. Moessner, and C. Castelnovo, Emergent Coulombic criticality and Kibble-Zurek scaling in a topological magnet, Phys. Rev. B 92, 075142 (2015).

[41] G. Biroli, L. F. Cugliandolo, and A. Sicilia, Kibble-Zurek mechanism and infinitely slow annealing through critical points, Phys. Rev. E 81, 050101(R) (2010).

[42] P. C. Hohenberg and B. I. Halperin, Theory of dynamic critical phenomena, Rev. Mod. Phys. 49, 435 (1977).

[43] P. W. Anderson, Ordering and antiferromagnetism in ferrites, Phys. Rev. 102, 1008 (1956).

[44] M. J. Harris, S. T. Bramwell, D. F. McMorrow, T. Zeiske, and K. W. Godfrey, Geometrical Frustration in the Ferromagnetic Pyrochlore $\mathrm{Ho}_{2} \mathrm{Ti}_{2} \mathrm{O}_{7}$, Phys. Rev. Lett. 79, 2554 (1997).

[45] S. T. Bramwell and M. J. P. Gingras, Spin ice state in frustrated magnetic pyrochlore materials, Science 294, 1495 (2001).

[46] R. Moessner and A. R. Ramirez, Geometrical frustration, Phys. Today $\mathbf{5 9}$ (2), 24 (2006).

[47] C. Castelnovo, R. Moessner, and S. L. Sondhi, Magnetic monopoles in spin ice, Nature (London) 451, 42 (2008).
[48] C. W. Liu, A. Polkovnikov, and A. W. Sandvik, Dynamic scaling at classical phase transitions approached through nonequilibrium quenching, Phys. Rev. B 89, 054307 (2014).

[49] Z. Budrikis, K. L. Livesey, J. P. Morgan, J. Akerman, A Stein, S. Langridge, C. H. Marrows, and R. L. Stamps, Domain dynamics and fluctuations in artificial square ice at finite temperatures, New J. Phys. 14, 035014 (2012).

[50] J. Drisko, T. Marsh, and J. Cumings, Topological frustration of artificial spin ice, Nat. Commun. 8, 14009 (2017).

[51] C. Fan and F. Wu, Ising model with second-neighbor interaction. I. Some exact results and an approximate solution. Phys. Rev. 179, 560 (1969).

[52] G. M. Macauley, G. W. Paterson, Y. Li, R. Macedo, S. McVitie, and R. L. Stamps, Tuning magnetic order with geometry: Thermalization and defects in two-dimensional artificial spin ices, Phys. Rev. B 101, 144403 (2020).

[53] F. Y. Wu, Critical behavior of Two-Dimensional HydrogenBonded Antiferroelectrics, Phys. Rev. Lett. 22, 1174 (1969).

[54] C. Nisoli, Dumping topological charges on neighbors: Ice manifolds for colloids and vortices, New J. Phys. 16, 113049 (2014).

[55] D. Levis, L. F. Cugliandolo, L. Foini, and M. Tarzia, Thermal Phase Transitions in Artificial Spin Ice, Phys. Rev. Lett. 110, 207206 (2013).

[56] V. Kapaklis, U. B. Arnalds, A. Farhan, R. V. Chopdekar, A Balan, A. Scholl, L. J. Heyderman, and B. Hjörvarsson, Thermal fluctuations in artificial spin ice, Nat. Nanotechnol. 9, 514 (2014).

[57] L. Anghinolfi, H. Luetkens, J. Perron, M. G. Flokstra, O. Sendetskyi, A. Suter, T. Prokscha, P. M. Derlet, S. L. Lee, and L. J. Heyderman, Thermodynamic phase transitions in a frustrated magnetic metamaterial, Nat. Commun. 6, 8278 (2015).

[58] A. Farhan, C. F. Petersen, S. Dhuey, L. Anghinolfi, Q. H. Qin, M. Saccone, S. Velten, C. Wuth, S. Gliga, P. Mellado, M. J. Alava, A. Scholl, and S. van Dijken, Nanoscale control of competing interactions and geometrical frustration in a dipolar trident lattice, Nat. Commun. 8, 995 (2017).

[59] Y. Lao, F. Caravelli, M. Sheikh, J. Sklenar, D. Gardeazabal, J. D. Watts, A. M. Albrecht, A. Scholl, K. Dahmen, C. Nisoli, and P. Schiffer, Classical topological order in the kinetics of artificial spin ice, Nat. Phys. 14, 723 (2018).

[60] J. Sklenar, Y. Lao, A. Albrecht, J. D. Watts, C. Nisoli, G.-W. Chern, and P. Schiffer, Field-induced phase coexistence in an artificial spin ice, Nat. Phys. 15, 191 (2019).

[61] Y. Perrin, B. Canals, and N. Rougemaille, Extensive degeneracy, Coulomb phase and magnetic monopoles in artificial square ice, Nature (London) 540, 410 (2016).

[62] A. Farhan, M. Saccone, C. F. Petersen, S. Dhuey, R. V. Chopdekar, Y.-L. Huang, N. Kent, Z. Chen, M. J. Alava, T. Lippert, A. Scholl, and S. van Dijken, Emergent magnetic monopole dynamics in macroscopically degenerate artificial spin ice, Sci. Adv. 5, eaav6380 (2019). 